\title{
Serum exosomal microRNAs as novel biomarkers for hepatocellular carcinoma
}

\author{
Won Sohn ${ }^{1,4}$, Jonghwa Kim ${ }^{1,4}$, So Hee Kang ${ }^{1}$, Se Ra Yang ${ }^{1}$, Ju-Yeon Cho', Hyun Chin Cho ${ }^{2}$, Sang Goon Shim ${ }^{2}$ \\ and Yong-Han Paik ${ }^{1,3}$
}

Recent studies have shown that circulating microRNAs are a potential biomarker in various types of malignancies. The aim of this study was to investigate the feasibility of using serum exosomal microRNAs as novel serological biomarkers for hepatocellular carcinoma (HCC) in patients with chronic hepatitis B (CHB). We measured the serum exosomal microRNAs and serum circulating microRNAs in patients with CHB $(n=20)$, liver cirrhosis $(\mathrm{LC})(n=20)$ and HCC $(n=20)$. Serum exosomal microRNA was extracted from $500 \mu \mathrm{l}$ of serum using an Exosome RNA Isolation kit. The expression levels of microRNAs were quantified by real-time PCR. The expression levels of selected microRNAs were normalized to Caenorhabditis elegans microRNA (Cel-miR-39). The serum levels of exosomal miR-18a, miR-221, miR-222 and miR-224 were significantly higher in patients with HCC than those with CHB or LC $(\boldsymbol{P}<0.05)$. Further, the serum levels of exosomal miR-101, miR-106b, miR-122 and miR-195 were lower in patients with HCC than in patients with CHB $(P=0.014, P<0.001, P<0.001$ and $P<0.001$, respectively). There was no significant difference in the levels of miR-21 and miR-93 among the three groups. Additionally, the serum levels of circulating microRNAs showed a smaller difference between HCC and either CHB or LC. This study suggests that serum exosomal microRNAs may be used as novel serological biomarkers for HCC.

Experimental \& Molecular Medicine (2015) 47, e184; doi:10.1038/emm.2015.68; published online 18 September 2015

\section{INTRODUCTION}

MicroRNAs or miRs are small, non-coding RNA molecules that modify posttranscriptional gene regulation. ${ }^{1}$ They can suppress the protein expression of specific mRNAs through either degradation or translational inhibition. ${ }^{2}$ Alterations of microRNAs have been reported in a variety of human diseases, including malignancies. ${ }^{3}$

Recently, the role of microRNA has been a major topic in cancer research. MicroRNAs in human cancer are associated with cell proliferation, cell metabolism, genomic instability, tumor invasion, metastasis, angiogenesis, apoptosis and immune responses. ${ }^{4}$ Clinical applications of microRNA have been tried in a variety of malignancies, such as lung cancer, breast cancer, hematologic malignancies, brain tumor, ovarian cancer and prostate cancer. In particular, microRNA has been studied as a biomarker for histological classification, disease prognosis and clinical response, as well as the diagnosis of cancer. ${ }^{5}$ Because of the ease and repeatability of sample collection, serum and plasma microRNA are being actively investigated as noninvasive biomarkers in human cancers. The role of miRNA in the diagnosis and therapy of hepatocellular carcinoma (HCC) and other malignancies has been studied. The expression of multiple microRNAs was increased or decreased in HCC tumor tissues. In addition, several circulating microRNAs have been reported as candidates for biomarkers associated with HCC. ${ }^{6,7}$

Exosomes are small membranous vesicles that originate from internal multivesicular bodies. Exosomes have been found in body fluids such as plasma, urine, saliva, breast milk and synovial fluid. ${ }^{8}$ Exosomes contain cell-specific protein, mRNA and microRNA. ${ }^{9}$ Recent studies showed that exosomal microRNA is stable in blood because exosomes have a protective function against degradation from enzymes, such as RNAse. ${ }^{10,11}$ Additionally, exosomal microRNA has a potential role as a diagnostic biomarker in patients with lung and ovarian cancer. ${ }^{12,13}$ However, the role of exosomal microRNA in HCC has not yet been reported.

${ }^{1}$ Department of Medicine, Samsung Medical Center, Sungkyunkwan University School of Medicine, Seoul, Korea; ${ }^{2}$ Department of Medicine, Samsung Changwon Hospital, Sungkyunkwan University School of Medicine, Changwon, Korea and ${ }^{3}$ Department of Health Science and Technology, Samsung Advanced Institute for Health Science and Technology, Sungkyunkwan University, Seoul, Korea

${ }^{4}$ These authors contributed equally to this work.

Correspondence: Dr Y-H Paik, Department of Medicine, Samsung Medical Center, Sungkyunkwan University School of Medicine, 81 Irwon-Ro, Gangnam-gu, Seoul 135-710, Korea.

E-mail: yh.paik@skku.edu

Received 4 December 2014; revised 17 June 2015; accepted 19 June 2015 
The aim of this study was to investigate the feasibility of using serum exosomal microRNAs as novel serological biomarkers for HCC. We measured the expression levels of serum exosomal microRNAs in patients with hepatitis B virus (HBV)-related chronic hepatitis, liver cirrhosis (LC) and HCC. The expression of serum exosomal microRNAs in HCC patients were compared with those of patients with chronic hepatitis $\mathrm{B}(\mathrm{CHB})$ or LC.

\section{MATERIALS AND METHODS}

\section{Patients}

From June 2012 to July 2013, 60 patients at Samsung Medical Center were enrolled in this study. The patients were all hepatitis B surface antigen positive and were divided into three groups: CHB $(n=20)$, LC $(n=20)$, and HCC $(n=20)$. Each group had the same gender ratio (women; $n=6$, and men; $n=14$ ). Platelet count, prothrombin time, serum albumin, total bilirubin, aspartate aminotransferase, alanine aminotransferase, HBV DNA level, hepatitis B envelope antigen, alpha-fetoprotein and abdominal ultrasonogram were checked in the enrolled patients. LC was evaluated by an imaging study such as an ultrasonogram or computed tomography. The presence of cirrhosis was defined as a coarse change of the hepatic parenchyma and surface nodularity with or without splenomegaly, ascites and varices. Patients with HCC were all treated with surgical resection. HCC was diagnosed based on the histological findings in the resected specimen. The Barcelona Clinic Liver Cancer staging system was used to evaluate the tumor stage. Serum exosomal microRNAs were analyzed in all patients. The serum exosomal microRNAs in patients with HCC were measured before surgical resection. This study was approved by the institutional review board of the Samsung Medical Center. Informed consent was received from all patients.

\section{Isolation of exosomes from serum and purification of exosomal RNAs}

Peripheral blood samples from the subjects were collected and centrifuged at $1300 \mathrm{~g}$ for $10 \mathrm{~min}$ at $4^{\circ} \mathrm{C}$. The supernatants were transferred to fresh tubes and stored at $-80^{\circ} \mathrm{C}$ until analysis. ExoQuick Exosome Precipitation Solution (System Biosciences, Mountain View, CA, USA) was used to isolate exosomes from the serum. The isolation, purification and elution of exosomal RNA were performed according to the manufacturer's protocol. First, $500 \mu \mathrm{l}$ of serum was mixed with $120 \mu$ l ExoQuick solution and then kept at $4{ }^{\circ} \mathrm{C}$ for $30 \mathrm{~min}$. The mixed solution was centrifuged at 13000 r.p.m. for $2 \mathrm{~min}$. The exosome pellets were obtained after the supernatants were removed. Three hundred and fifty microliters of phenol-free lysis buffer was added to the exosome pellets. Twenty-five picomoles of synthetic Caenorhabditis elegans miRNA (Cel-miR-39, Ambion, Austin, TX, USA) was added to each sample as the internal control. ${ }^{14}$ To purify the exosomal RNA, $200 \mu$ l of $100 \%$ ethanol was added to the exosomal preparations. Thereafter, the preparations were transferred to a spin column and centrifuged at 13000 r.p.m. for $1 \mathrm{~min}$. We discarded the flow-through and added $200 \mu \mathrm{l}$ of wash buffer; then the preparations were centrifuged at 13000 r.p.m. for $1 \mathrm{~min}$. The addition of the wash buffer and the centrifugation were repeated once. For the elution of exosomal RNA, $30 \mu$ of elution buffer was added directly to the membrane in the spin column. Thereafter, the centrifugation was performed at 2000 r.p.m. for $2 \mathrm{~min}$ and 13000 r.p.m. for $1 \mathrm{~min}$.

\section{Western blotting analysis}

To identify the isolation of the exosomes from the serum, western blotting analysis was performed for the cluster of differentiation 63 (CD63) and CD9, which are enriched in exosomes, and for calnexin, which is an integral protein of the endoplasmic reticulum and is not expressed in the exosome. The expression of CD63, CD9 and calnexin in the exosome from the serum of enrolled patients was compared with that of healthy people and the lysates of Huh-7 cells. Isolated exosomal pellets from the serum were washed in phosphate-buffered saline and treated with a lysis buffer containing RIPA lysis buffer. Serum exosomal preparations and Huh-7 cells were incubated with rabbit polyclonal anti-human (CD63) immunoglobulin G (IgG), rabbit polyclonal anti-human CD9 IgG (System Biosciences) and rabbit polyclonal anti-human calnexin (Cell signaling Technology, Beverly, MA, USA), followed by goat anti-rabbit horseradish peroxidase (System Biosciences).

\section{Isolation of serum RNA}

The total RNA, including microRNA, was extracted from serum using the miRNeasy Serum/Plasma Kit (Qiagen, Hilden, Germany). Two hundred microliters of serum was mixed with denaturing buffer in the volumes described in the manufacturer's protocols. The homogenate was incubated at room temperature for $5 \mathrm{~min}$. Then $25 \mathrm{fmol}$ of synthetic Cel-miR-39 (Ambion) was spiked into the mixture. Subsequently, the manufacturer's protocols were followed for RNA extraction. Total RNA was eluted into $14 \mu \mathrm{l}$ of nuclease-free water. The RNA concentration was measured using a NanoDrop ND-1000 spectrophotometer (NanoDrop Technologies, Wilmington, DE, USA).

\section{Measurement of serum exosomal microRNA levels and serum circulating microRNA levels using real-time quantitative PCR}

Ten nanograms of total RNA was reverse transcribed using the TaqMan microRNA Reverse Transcription Kit (Applied Biosystems, Carlsbad, CA, USA) and microRNA-specific stem-loop primers (part of the TaqMan microRNA Assay Kit; Applied Biosystems). The above mixture was incubated at $37^{\circ} \mathrm{C}$ for $60 \mathrm{~min}, 94^{\circ} \mathrm{C}$ for $5 \mathrm{~min}$ and $4{ }^{\circ} \mathrm{C}$ for $10 \mathrm{~min}$. Among the numerous target miRNAs, we examined 10 miRNAs that were increased or decreased in HCC tissue in previous studies. ${ }^{15-24}$ To measure serum exosomal microRNAs, miR-18a, -21, $-93,-106 \mathrm{~b},-221,-222$ and -224 were selected as upregulated microRNAs, and miR-101, 122 and 195 were selected as downregulated microRNAs. The measurement of serum circulating microRNAs was performed for six microRNAs (miR-21, -221, -222, $-224,-101$ and -195). The real-time quantitative PCR for microRNAs was conducted according to the TaqMan miRNA assay protocol (Applied BioSystems). First, $1.5 \mu \mathrm{RT}$ product was combined with the TaqMan Universal PCR master mix and $0.5 \mu$ l probe mix of the TaqMan MicroRNA Assay. The total volume of the mixture product for PCR was $10 \mu$ l. The incubation of the mixture product was carried out at $95^{\circ} \mathrm{C}$ for $10 \mathrm{~min}$, followed by 40 cycles of $94^{\circ} \mathrm{C}$ for $15 \mathrm{~s}$ and $60^{\circ}$ $\mathrm{C}$ for $1 \mathrm{~min}$. Real-time quantitative PCR was performed using the 7900HT Fast Real-Time PCR System (Applied Biosystems), and the results were analyzed using the RQ manager software (Applied Biosystems). The amplification plot reflecting the fluorescent signal at each cycle was determined based on the threshold cycle $(\mathrm{Ct})$ values for each sample. The average of the $\mathrm{Ct}$ value was calculated after the PCRs were run in duplicate for each sample. The Cel-miR-39 value from the duplicate was used as the internal control. ${ }^{13,14}$ The relative 
gene expression values for the target microRNA were normalized to Cel-miR-39 and calculated using the $2^{-\Delta \Delta C T}$ method..$^{25,26}$

\section{Statistical analysis}

All results except the microRNA value were described as the median with a range. The microRNA value is expressed as the mean \pm s.e.m. The Mann-Whitney $U$-test was used to analyze differences between two groups (CHB vs HCC and LC vs HCC). The Spearman correlation coefficient $(r)$ was used to evaluate the correlation of miRNA expression between serum exosomal and circulating miRNAs. All data were statistically analyzed using SPSS (Statistical Package for the Social Sciences) for Windows release 18.0 (SPSS Inc., Chicago, IL, USA). Statistical significance was considered positive when $P$-value was $<5 \%$ in the two-sided $t$-test.

\section{RESULTS}

\section{Clinical characteristics of the subjects}

Table 1 shows the clinical characteristics of the subjects in this study. All patients were hepatitis B surface antigen positive. Each group (CHB, LC and HCC) consisted of 20 persons, respectively. The gender ratio was the same in each group (14 men and 6 women). The HCC group had a lower platelet count than the $\mathrm{CHB}$ group but a higher platelet count than the LC group. The serum aspartate aminotransferase, alanine aminotransferase and alpha-fetoprotein levels of the HCC group were higher than those of the CHB or LC groups. The median size of the HCC group was $4.7(1.5-13.0) \mathrm{cm}$. The number of tumors in the HCC group was 1 tumor in 15 patients, 2 in 5 patients, and $\geqslant 3$ in 3 patients. The Barcelona Clinic Liver Cancer stage of HCC was very early in 2 patients, early in 14 patients and intermediate in 4 patients. Microvascular invasion of the tumor was shown in 16 patients $(80 \%)$.

\section{Identification of serum exosomes}

Western blotting analysis was performed to confirm the isolation of exosomes from serum (Figure 1). CD63, CD9 and calnexin were used to identify serum exosomes. We compared the expression of CD63, CD9 and calnexin in isolated exosomal pellets from the sera of patients with that of the healthy controls and the lysates from the Huh-7 cells. The expression of CD63 and CD9 was observed in the sera of patients and healthy controls, whereas calnexin (a negative marker of exosomes) was only expressed in the Huh-7 cells, which indicates that the exosomes had been adequately purified.

\section{Expression level of serum exosomal miRNAs}

The differences in the levels of 10 serum exosomal microRNAs among the CHB, LC and HCC groups are demonstrated in Figure 2. The distribution of upregulated or downregulated exosomal miRNAs in HCC patients is demonstrated in Figures 3 and 4, respectively. Compared with the CHB group, the levels of serum exosomal miR-18a, miR-221, miR-222 and miR-224 were significantly increased in the HCC group $(P=0.004, P=0.001, P=0.001$ and $P=0.001$, respectively;

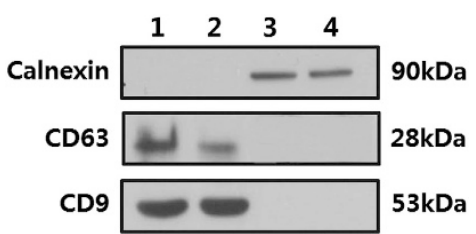

Figure 1 Expression of CD63, CD9 and calnexin by western blotting analysis. Lane 1: isolated exosomal pellets from the serum of healthy humans (positive control), lane 2: isolated exosomal pellets from the serum of patients, and lanes 3 and 4: Huh-7 cell lysates.

Table 1 Clinical characteristics of the subjects $(N=60)$

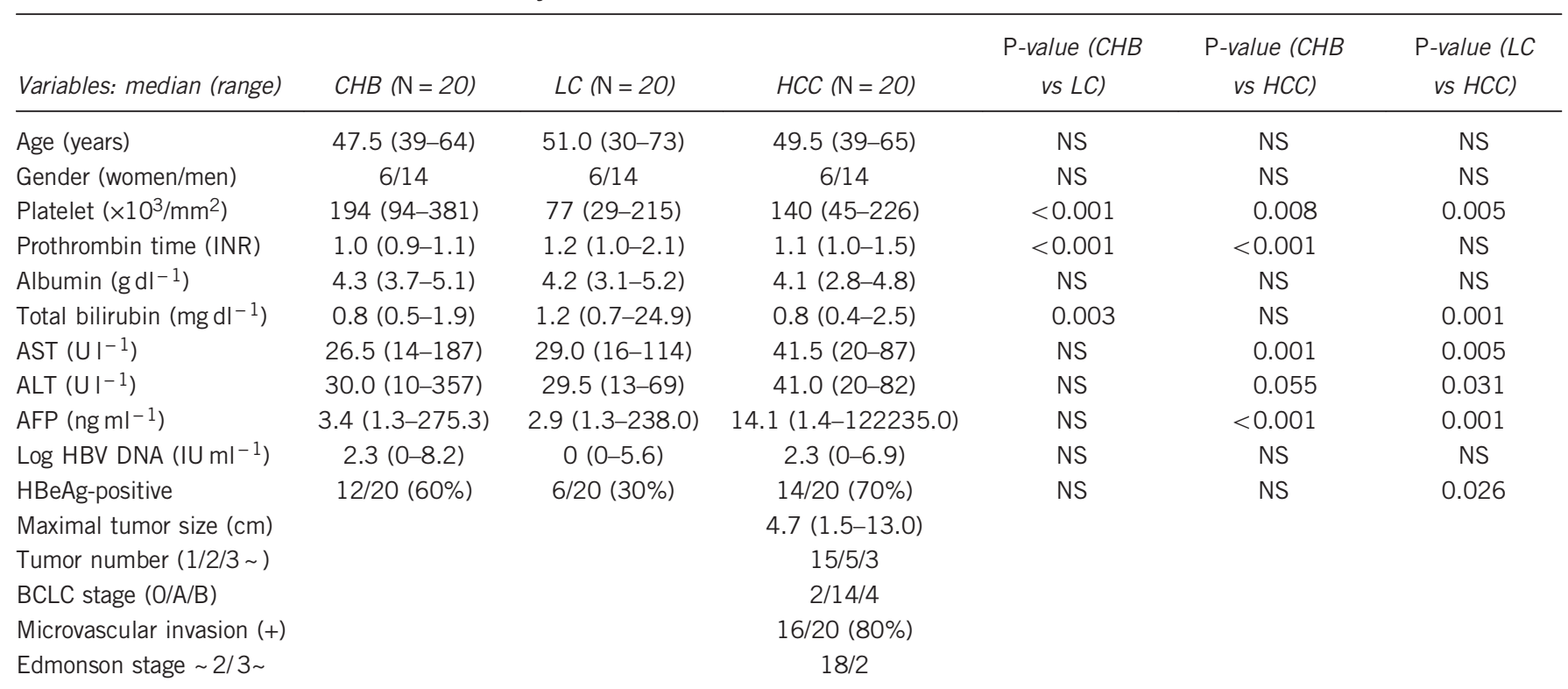

Abbreviations: AFP, alpha-fetoprotein; ALT, alanine aminotransferase; AST, aspartate aminotransferase; BCLC, Barcelona Clinic Liver Cancer; CHB, chronic hepatitis B; $\mathrm{HBeAg}$, hepatitis B envelope antigen; HBV, hepatitis B virus; HCC, hepatocellular carcinoma; INR, international normalized ratio; LC, liver cirrhosis; NS, not significant. 


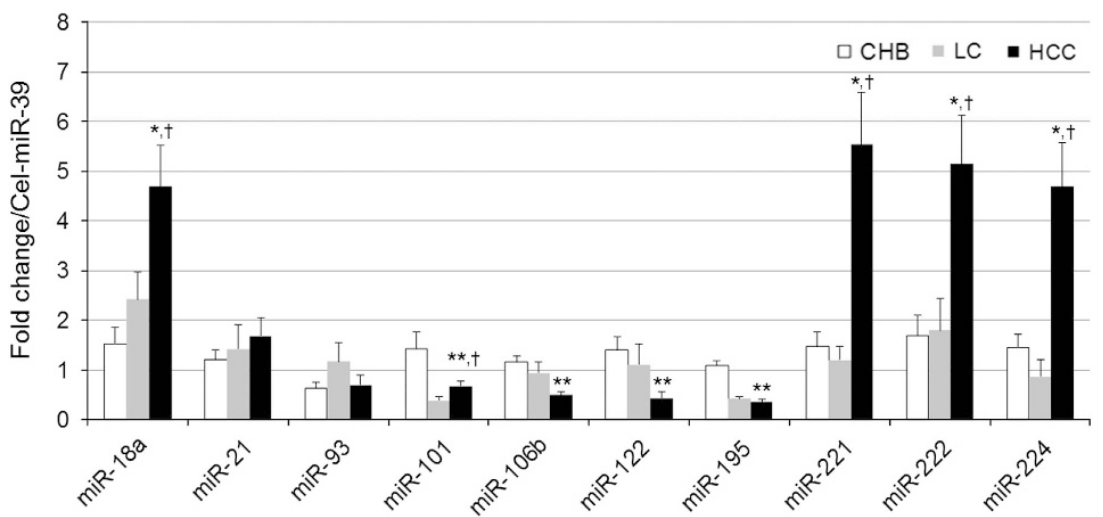

Figure 2 Serum exosomal microRNAs from patients with chronic hepatitis B (CHB), liver cirrhosis (LC) and hepatocellular carcinoma (HCC). The levels of serum exosomal microRNA were measured by real-time quantitative PCR. The values of the relative gene expression for target microRNA were normalized to Cel-miR-39 and calculated using the $2^{-\Delta \Delta C T}$ method. *The level of serum exosomal microRNA was increased in the HCC group compared with the CHB group $(P<0.05)$. **The level of serum exosomal microRNA was decreased in the HCC group compared with the $\mathrm{CHB}$ group $(P<0.05)$. ${ }^{\dagger}$ The level of serum exosomal microRNA was increased in the HCC group compared with the LC group $(P<0.05)$. The values are expressed as the mean \pm s.e.m.

Figures 2 and 3). However, the levels of serum exosomal miR-101, miR-106b, miR-122 and miR-195 were significantly lower in the HCC group than in the CHB group $(P=0.014$, $P<0.001, P<0.001$ and $P<0.001$, respectively; Figures 2 and 4$)$. There was no difference in the levels of serum exosomal miR-21 and miR-93 between the HCC and CHB groups ( $P=0.925$ and $P=0.355$, respectively).

The levels of serum exosomal miR-18a, miR-221, miR-222 and miR-224 were significantly higher in patients with HCC than in those with LC $(P=0.028, P<0.001, P<0.001$ and $P<0.001$, respectively). Meanwhile, the level of miR-101 was lower in patients with HCC than in those with LC $(P=0.008)$ However, there was no difference in the levels of serum exosomal miR-21, miR-93, miR-106b, miR-122 and miR-195 between the HCC and LC groups $(P=0.414, P=0.081$, $P=0.602, P=0.883$ and $P=0.108$, respectively).

\section{Expression level of serum circulating microRNAs}

The differences in the levels of six serum circulating miRNAs among the CHB, LC and HCC groups are demonstrated in Figure 5. Compared with the LC group, the levels of serum miR-21, miR-221, miR-222 and miR-224 were significantly increased in the HCC group $(P=0.001, P=0.022, P<0.001$ and $P<0.001$, respectively). However, there was no significant difference in the serum levels of miR-101 and miR-195 between the HCC and LC groups $(P=0.725$ and $P=0.428$, respectively). Although the levels of serum miR-21 and miR-221 were generally higher in the HCC group compared with the $\mathrm{CHB}$ group, this difference did not reach statistical significance $(P=0.088$ and $P=0.053$, respectively). There was no difference in serum miR-101, miR-195, miR-222 and miR-224 between the HCC and $\mathrm{CHB}$ groups $(P=0.113$, $P=0.379, P=0.692$ and $P=0.218$, respectively).

The correlation of miRNA expression (miR-21, miR-101, miR-195, miR-221, miR-222 and miR-224) between serum exosomal and circulating miRNAs was investigated in each group. A high correlation coefficient was observed in miR-21 in CHB $(r=0.636, P=0.048), \operatorname{miR}-221$ in LC $(r=0.770$, $P=0.009)$, miR-222 in HCC $(r=0.508, P=0.022)$ and miR-224 in HCC $(r=0.547, P=0.012)$ (Figure 5 and Table 2).

\section{DISCUSSION}

Recently, there has been a great interest in the roles of microRNA in the field of cancer research. Alterations of cancer tissue and circulating microRNAs have been shown in patients with HCC. ${ }^{27}$ However, there have been no reports on serum exosomal microRNAs in HCC. We investigated the levels of serum exosomal microRNAs in HCC patients and compared them with the levels observed in $\mathrm{CHB}$ or LC patients. The levels of serum exosomal miR-18a, miR-221, miR-222 and miR-224 were significantly higher in patients with HCC than in patients with CHB or LC. Meanwhile, the levels of miR-101, miR-106b, miR-122 and miR-195 were lower in HCC than those with CHB. Our data suggest that serum exosomal microRNA may be used as a novel biomarker for HCC.

MicroRNA is one of the short non-coding RNAs, which also include PIWI-interacting RNAs and small nucleolar RNAs. ${ }^{28}$ The first report of microRNA in cancer showed the deletion of a gene cluster that contained miR-15 and miR-16 in patients with chronic lymphocytic leukemia. ${ }^{29}$ MicroRNAs are involved in the development of cancer by downregulating tumorsuppressor genes or upregulating oncogenes. Additionally, the dysregulation of microRNAs in cancer is associated with cancer proliferation, tumor invasion, metastasis, sustained angiogenesis and inhibition of apoptosis. ${ }^{30}$

The studies to identify the actions of microRNAs in the development or progression of HCC have been reported. The overexpression of miR-18a induced the proliferation of HCC cells and reduced the level of estrogen receptor- $\alpha .{ }^{31}$ MiR-221 contributed to hepatocarcinogenesis by dysregulating DNA damage-inducible transcript 4 and targeting the Bmf gene relevant to apoptosis in HCC. ${ }^{32,33}$ The overexpression of 

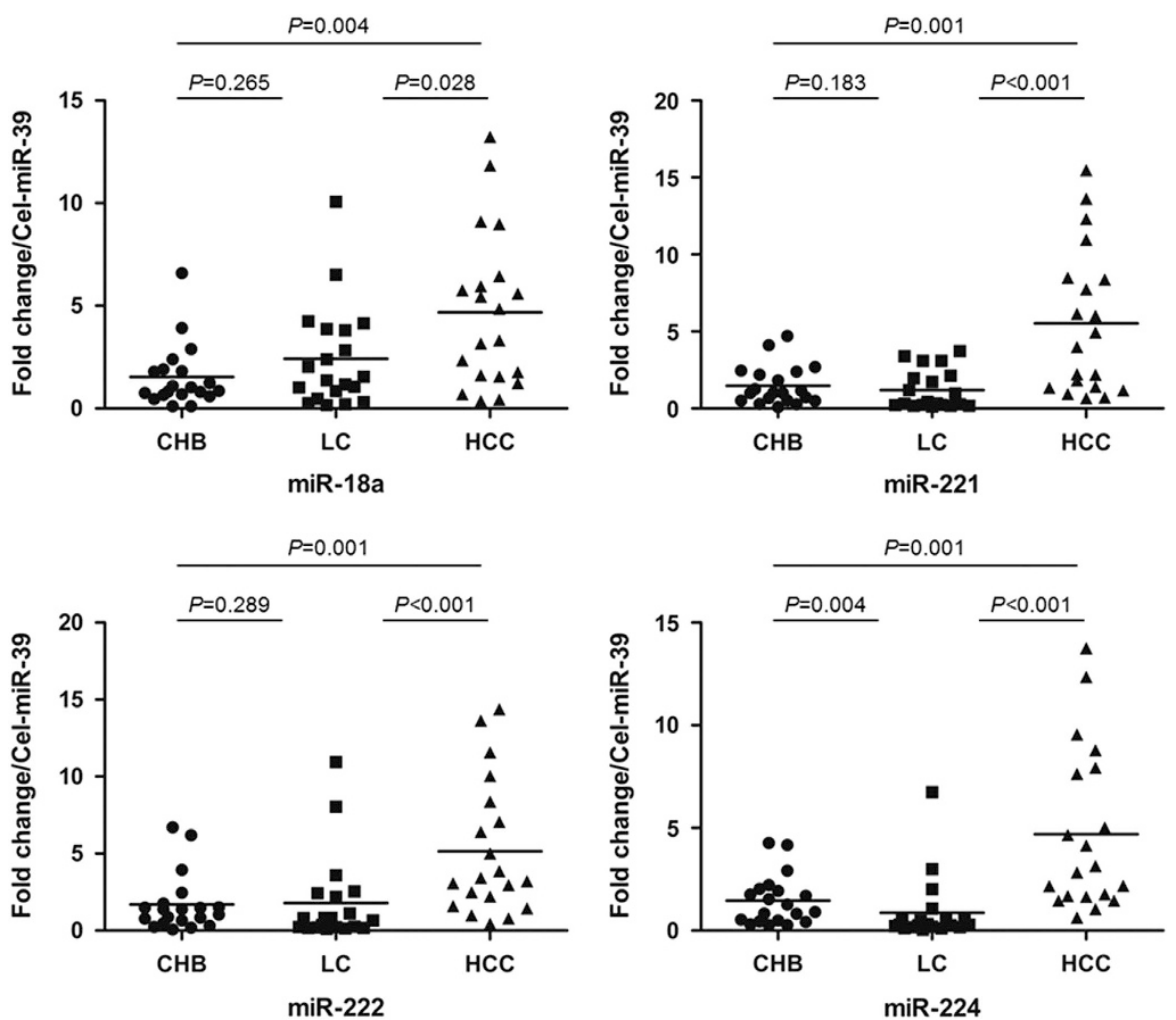

Figure 3 The distribution of upregulated exosomal microRNAs (miR-18a, -221, -222 and -224) in patients with CHB, LC and HCC. Each serum exosomal microRNA was compared between two groups (CHB vs HCC, CHB vs LC and HCC vs LC). The levels of serum exosomal microRNA were measured by real-time quantitative PCR. The values of relative gene expression for target microRNA were normalized to Cel-miR-39 and calculated using the $2^{-\Delta \Delta C T}$ method. $P<0.05$ was considered statistically significant. CHB, chronic hepatitis B; LC, liver cirrhosis; HCC, hepatocellular carcinoma.

miR-222 was associated with cell migration in HCC through enhanced AKT signaling. ${ }^{34}$ Downregulated miR-101 inhibited apoptosis and induced tumorigenicity in HCC. ${ }^{16}$ MiR-122 acted as a tumor suppressor and regulated the intrahepatic metastasis of HCC. ${ }^{35}$ MiR-195 suppressed tumorigenicity and regulated the G1/S transition of HCC cells. ${ }^{36}$

Alterations of numerous microRNAs have been demonstrated in HCC. Some microRNAs were upregulated in HCC tumor tissue, whereas others were downregulated in HCC compared with a healthy liver. ${ }^{6,27}$ In addition, several reports have shown that circulating microRNA can be a possible biomarker for the diagnosis of HCC. Qu et al..$^{37}$ reported that the serum level of miR-199a level was higher in HCC patients than in chronic liver disease or healthy controls, whereas the serum levels of miR-16 and miR-195 were significantly lower in HCC than in either patients with chronic liver disease or healthy controls. Xu et al..$^{38}$ demonstrated that serum miR-21, miR-122 and miR-223 were significantly increased in patients with HCC.

Exosomes are membrane vesicles that are sized between 40 and $1000 \mathrm{~nm}$ and are released from a variety of cells. ${ }^{39}$ They contain microRNA, mRNA and proteins. ${ }^{10}$ Recently, serum and plasma microRNAs have been studied as valuable biomarkers in cancer for the following reasons. First, RNAs inside the exosome are protected from degradation enzymes in the blood, whereas naked RNAs are rapidly degraded. ${ }^{40}$ Tanaka et al. ${ }^{41}$ revealed that serum exosomal miR-21 was significantly higher in human esophageal squamous cell carcinoma than in benign diseases and that the level of serum exosomal miRNA was higher than that of serum non-exosomal microRNA. Second, exosomes reflect their tissue of origin because they can be immune-isolated using an antibody to a tissue-specific protein on the membrane surface. ${ }^{9}$ In fact, serum exosomal microRNAs have been correlated to tumor-derived microRNAs and have shown great potential as diagnostic biomarkers for ovarian and lung cancer. ${ }^{12,42}$

In the present study, the serum levels of 10 serum exosomal microRNAs (miR-18a, -21, -93, -101, -106b, -122, -195, -221, -222 and -224) were investigated in patients with HCC. Compared with previous studies, our results are mostly consistent with those obtained for tissue microRNA in patients with HBV-related HCC. Connolly et al. ${ }^{15}$ showed that miR-18a, miR-21, miR-93 and miR-106b of human HCC tissue with HBV infection were increased compared with the non-tumor liver tissue of the controls, whereas miR-122a was decreased in HCC tissue. Su et al. ${ }^{16}$ reported that miR-18, miR-93, miR-222 and miR-224 were increased in human HCC tissue but that miR-101 and miR-195 were decreased in HCC 

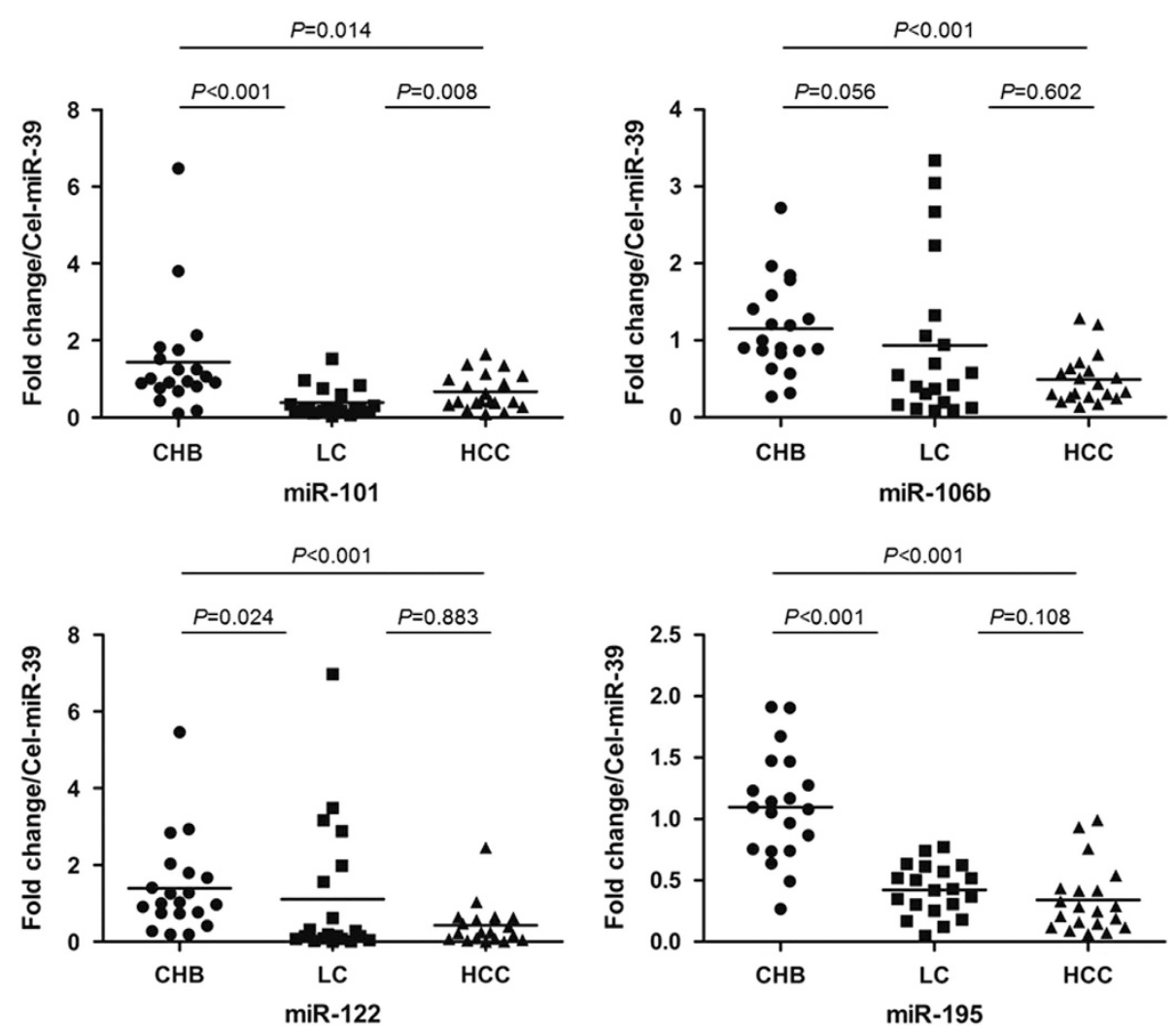

Figure 4 The distribution of downregulated exosomal microRNAs (miR-101, -106b, -122 and -195) in patients with CHB, LC and HCC. Each serum exosomal microRNA was compared between two groups (CHB vs HCC, CHB vs LC and HCC vs LC). The levels of serum exosomal microRNA were measured by real-time quantitative PCR. The values of relative gene expression for target microRNA were normalized to Cel-miR-39 and calculated using the $2^{-\Delta \Delta C T}$ method. $P<0.05$ was considered statistically significant. $C H B$, chronic hepatitis B; LC, liver cirrhosis; HCC, hepatocellular carcinoma.

tissue compared with normal liver tissue. Wong et al. ${ }^{24}$ showed that miR-31, miR-221 and miR-222 were upregulated in HCC tissue compared with adjacent non-tumoral liver tissue and that miR-122a, miR-126 and miR-223 were downregulated in HCC tissue. In our study, serum exosomal miR-106b was downregulated in HCC, which contrasts with the results of the study of tissue microRNA by Connolly et al. ${ }^{15}$

Although the expression of tissue microRNA differs between HCC and non-HCC, clinical application is not easy because a liver tissue biopsy is invasive and because it is difficult to repeat the procedure. Therefore, serum or plasma circulating microRNA has been studied as a potential biomarker for malignancies. ${ }^{43}$ Several circulating microRNAs were investigated as a useful biomarker for HCC. Xu et al. ${ }^{38}$ showed that serum circulating miR-21, miR-122 and miR-223 were significantly increased in patients with HCC. Qu et al. ${ }^{37}$ reported that serum miR-16 and miR-199a were decreased in patients with HCC compared with CHB or control subjects. Li et al..$^{44}$ showed that the serum levels of miR-21, miR-221, miR-222 and miR-224 were higher in patients with HCC than in healthy controls. Moreover, elevated miR-221 was associated with the stage and the prognosis of HCC. Qi et al. ${ }^{45}$ demonstrated that serum miR-122, miR-222 and miR-223 were upregulated in patients with HCC compared with healthy subjects but that miR-21 was downregulated in HCC patients.

In the present study, we investigated the serum levels of exosomal microRNAs in patients with HCC compared with chronic hepatitis or LC. Similar to the results of a previous study on circulating microRNAs, the serum levels of miR-221, miR-222 and miR-224 were higher in patients with HCC than in those with CHB or LC. The level of exosomal miR-122 was downregulated in HCC, unlike in the study by Qi et al. ${ }^{45}$ However, this downregulation of serum exosomal miR-122 is consistent with the results of another microRNA study using HCC tissue. $15,17,20,21,23$ There was no significant difference in the level of miR-21 between HCC and non-HCC patients, unlike other previous reports of circulating miRNA. In this study, we measured the levels of serum circulating microRNAs in addition to those of serum exosomal microRNAs. The expression pattern of serum circulating microRNAs (that is, miR-221, miR-222 and miR-224) between the HCC and LC groups was similar to that of serum exosomal microRNAs. However, the difference of miRNAs levels between the groups was smaller in the serum itself than in serum exosomes. Additionally, unlike serum exosomal microRNA, serum circulating microRNAs did not show a difference between the HCC 


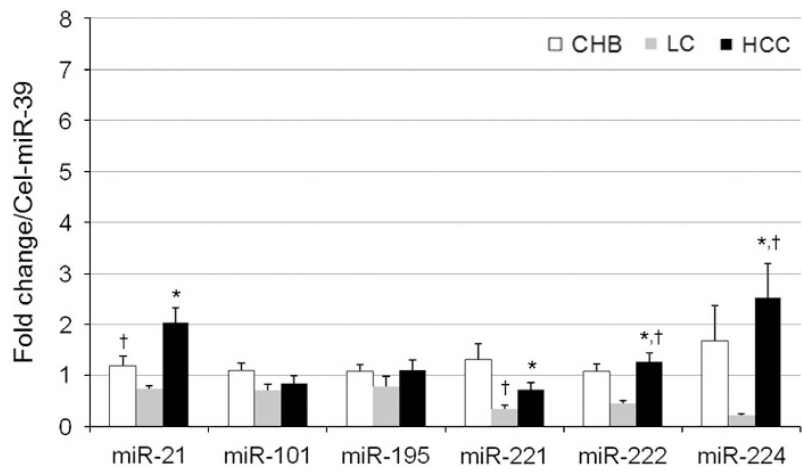

Figure 5 Serum circulating microRNAs in chronic hepatitis B (CHB), liver cirrhosis (LC) and hepatocellular carcinoma (HCC). The levels of serum circulating microRNA were measured by real-time quantitative PCR. The values of the relative gene expression for target microRNA were normalized to Cel-miR-39 and calculated using the $2^{-\Delta \Delta C T}$ method. *The level of serum microRNA was increased in the LC group compared with the CHB group $(P<0.05)$. There was no significant difference in the level of serum circulating microRNA between the $\mathrm{CHB}$ and HCC groups. The value was expressed as the mean \pm s.e.m. The correlation of microRNA expression between serum exosomal and circulating microRNAs was investigated in each group. ${ }^{\dagger} A$ high correlation coefficient was observed in miR-21 in CHB ( $r=0.636, P=0.048)$, miR-221 in LC $(r=0.770, P=0.009)$, miR-222 in HCC $(r=0.508, P=0.022)$ and miR-224 in HCC $(r=0.547, P=0.012)$.

and $\mathrm{CHB}$ groups. These findings indicate that the level of serum exosomal microRNAs is better for distinguishing HCC from $\mathrm{CHB}$ or LC compared with the level of serum circulating microRNAs.

This study has several limitations. First, the relationship between serum exosomal microRNA and tissue microRNA was not investigated. We did not analyze tissue microRNAs because a liver biopsy was not done. Realistically, it is not easy to perform a liver biopsy because it may cause potential complications, such as bleeding. Although further research to compare the expression of tissue microRNAs and serum exosomal microRNAs in patients with HCC is required, our study was able to demonstrate the potential role of serum exosomal microRNAs as a biomarker for HCC. Second, this study did not include healthy subjects as controls. HCC is a cancer that arises in patients with risk factors, such as chronic HBV or HCV infection, alcohol abuse, LC and aflatoxin exposure. Chronic HBV infection is the most common etiology of HCC. ${ }^{46}$ In Korea, chronic HBV infection is the most common cause of HCC, and approximately $70-80 \%$ of HCC cases are due to chronic HBV infection. The risk of HCC development is approximately 100 times greater in patients with chronic hepatitis B than it is in patients without hepatitis B. Additionally, the risk of HCC is increased in patients with LC. However, it has been demonstrated that the HCC occurrence in healthy people without any of these risks is very rare. ${ }^{47}$ Therefore, in this study, we included patients with chronic hepatitis B and HBV-related LC as control groups for HCC. Third, the levels of serum exosomal microRNA in patients with HCC did not
Table 2 Correlation of microRNA expression between serum exosomal and circulating microRNAs in each group

\begin{tabular}{|c|c|c|c|c|c|c|}
\hline & \multicolumn{2}{|c|}{$\mathrm{CHB}$} & \multicolumn{2}{|c|}{$L C$} & \multicolumn{2}{|c|}{$\mathrm{HCC}$} \\
\hline & $r$ & P-value & $r$ & P-value & $r$ & P-value \\
\hline miR-21 & 0.636 & 0.048 & 0.176 & 0.627 & -0.205 & 0.387 \\
\hline miR-101 & -0.055 & 0.881 & -0.217 & 0.726 & 0.017 & 0.942 \\
\hline miR-195 & 0.345 & 0.328 & -0.479 & 0.162 & -0.227 & 0.336 \\
\hline miR-221 & 0.309 & 0.385 & 0.770 & 0.009 & 0.352 & 0.128 \\
\hline miR-222 & 0.600 & 0.067 & 0.042 & 0.907 & 0.508 & 0.022 \\
\hline miR-224 & 0.527 & 0.117 & 0.248 & 0.489 & 0.547 & 0.012 \\
\hline
\end{tabular}

Abbreviations: $\mathrm{CHB}$, chronic liver disease; HCC, hepatocellular carcinoma; LC, liver cirrhosis; miR, microRNA.

' $r$ ' is the correlation coefficient between serum exosomal and circulating microRNAs.

A $P$-value $<0.05$ is considered statistically significant.

show any correlation with tumor characteristics, such as tumor stage, the presence of microvascular invasion and tumor differentiation. These results seem to be due to the relatively low number of subjects. Further large-scaled studies clarifying the role of serum exosomal microRNAs in HCC are currently underway.

In conclusion, there was a significant difference in the levels of serum exosomal microRNAs in patients with HCC compared with patients with CHB or LC. This study suggests that serum exosomal microRNAs may be a novel serological biomarker for HCC.

\section{CONFLICT OF INTEREST}

The authors declare no conflict of interest.

\section{ACKNOWLEDGEMENTS}

This work was supported by funding from the National Research Foundation of Korea (NRF) grant funded by the Korea government (MEST) (No. 2012R1A1A2041980) (to YHP)

1 Bushati N, Cohen SM. microRNA functions. Annu Rev Cell Dev Biol 2007; 23: 175-205.

2 Bartel DP. MicroRNAs: genomics, biogenesis, mechanism, and function. Cell 2004; 116: 281-297.

3 Benhamou Y, Di Martino V, Bochet M, Colombet G, Thibault V, Liou A et al. Factors affecting liver fibrosis in human immunodeficiency virus-and hepatitis $C$ virus-coinfected patients: impact of protease inhibitor therapy. Hepatology 2001; 34: 283-287.

4 Ruan K, Fang X, Ouyang G. MicroRNAs: novel regulators in the hallmarks of human cancer. Cancer Lett 2009; 285: 116-126.

5 Nana-Sinkam SP, Croce CM. Clinical applications for microRNAs in cancer. Clin Pharmacol Ther 2013; 93: 98-104.

6 Borel F, Konstantinova P, Jansen PL. Diagnostic and therapeutic potential of miRNA signatures in patients with hepatocellular carcinoma. $J$ Hepatol 2012; 56: 1371-1383.

7 Giordano S, Columbano A. MicroRNAs: new tools for diagnosis, prognosis, and therapy in hepatocellular carcinoma? Hepatology 2013; 57: 840-847.

8 Lasser C, Exosomal RNA. as biomarkers and the therapeutic potential of exosome vectors. Expert Opin Biol Ther 2012; 12(Suppl 1): S189-S197.

9 Pant S, Hilton H, Burczynski ME. The multifaceted exosome: biogenesis, role in normal and aberrant cellular function, and frontiers for pharmacological and biomarker opportunities. Biochem Pharmacol 2012; 83: 1484-1494. 
10 Valadi H, Ekstrom K, Bossios A, Sjostrand M, Lee JJ, Lotvall JO. Exosomemediated transfer of mRNAs and microRNAs is a novel mechanism of genetic exchange between cells. Nat Cell Biol 2007; 9: 654-659.

11 Lima LG, Chammas R, Monteiro RQ, Moreira ME, Barcinski MA. Tumorderived microvesicles modulate the establishment of metastatic melanoma in a phosphatidylserine-dependent manner. Cancer Lett 2009; 283: 168-175.

12 Taylor DD, Gercel-Taylor C. MicroRNA signatures of tumor-derived exosomes as diagnostic biomarkers of ovarian cancer. Gynecol Oncol 2008; 110: 13-21.

13 Aroldi A, Lampertico P, Montagnino G, Passerini P, Villa M, Campise MR et al. Natural history of hepatitis $B$ and $C$ in renal allograft recipients. Transplantation 2005; 79: 1132-1136.

14 Mitchell PS, Parkin RK, Kroh EM, Fritz BR, Wyman SK, PogosovaAgadjanyan $\mathrm{E} L$ et al. Circulating microRNAs as stable blood-based markers for cancer detection. Proc Natl Acad Sci USA 2008; 105: 10513-10518.

15 Connolly E, Melegari M, Landgraf P, Tchaikovskaya T, Tennant BC, Slagle $B$ L et al. Elevated expression of the miR-17-92 polycistron and miR-21 in hepadnavirus-associated hepatocellular carcinoma contributes to the malignant phenotype. Am J Pathol 2008; 173: 856-864.

16 Su H, Yang JR, Xu T, Huang J, Xu L, Yuan Y et al. MicroRNA-101, downregulated in hepatocellular carcinoma, promotes apoptosis and suppresses tumorigenicity. Cancer Res 2009; 69: 1135-1142.

17 Meng F, Henson R, Wehbe-Janek H, Ghoshal K, Jacob ST, Patel T. MicroRNA-21 regulates expression of the PTEN tumor suppressor gene in human hepatocellular cancer. Gastroenterology 2007; 133: 647-658.

18 Wang Y, Lee AT, Ma JZ, Wang J, Ren J, Yang Y et al. Profiling microRNA expression in hepatocellular carcinoma reveals microRNA-224 up-regulation and apoptosis inhibitor-5 as a microRNA-224-specific target. J Biol Chem 2008; 283: 13205-13215.

19 Jiang J, Gusev Y, Aderca I, Mettler TA, Nagorney DM, Brackett DJ et al. Association of MicroRNA expression in hepatocellular carcinomas with hepatitis infection, cirrhosis, and patient survival. Clin Cancer Res 2008, 14: 419-427.

20 Ladeiro Y, Couchy G, Balabaud C, Bioulac-Sage P, Pelletier L, Rebouissou $S$ et al. MicroRNA profiling in hepatocellular tumors is associated with clinical features and oncogene/tumor suppressor gene mutations. Hepatology 2008; 47: 1955-1963.

21 Huang XH, Wang Q, Chen JS, Fu XH, Chen XL, Chen LZ et al. Bead-based microarray analysis of microRNA expression in hepatocellular carcinoma: miR-338 is downregulated. Hepatol Res 2009; 39: 786-794.

22 Murakami Y, Yasuda T, Saigo K, Urashima T, Toyoda H, Okanoue T et al. Comprehensive analysis of microRNA expression patterns in hepatocellular carcinoma and non-tumorous tissues. Oncogene 2006; 25: 2537-2545.

23 Gramantieri L, Ferracin M, Fornari F, Veronese A, Sabbioni S, Liu C G et al. Cyclin G1 is a target of miR-122a, a microRNA frequently downregulated in human hepatocellular carcinoma. Cancer Res 2007; 67: 6092-6099.

24 Wong QW, Lung RW, Law PT, Lai PB, Chan KY, To KF et al. MicroRNA-223 is commonly repressed in hepatocellular carcinoma and potentiates expression of Stathmin1. Gastroenterology 2008; 135: 257-269.

25 Livak KJ, Schmittgen TD. Analysis of relative gene expression data using real-time quantitative PCR and the 2(-Delta Delta $\mathrm{C}(\mathrm{T})$ ) Method. Methods 2001; 25: 402-408.

26 Schmittgen TD, Livak KJ. Analyzing real-time PCR data by the comparative C(T) method. Nat Protoc 2008; 3: 1101-1108.

27 Braconi C, Henry JC, Kogure T, Schmittgen T, Patel T. The role of microRNAs in human liver cancers. Semin Oncol 2011; 38: 752-763.

28 Esteller M. Non-coding RNAs in human disease. Nat Rev Genet 2011; 12 : 861-874.

29 Calin GA, Dumitru CD, Shimizu M, Bichi R, Zupo S, Noch E et al. Frequent deletions and down-regulation of micro- RNA genes miR15 and miR16 at $13 q 14$ in chronic lymphocytic leukemia. Proc Natl Acad Sci USA 2002 99: 15524-15529

30 Iorio MV, Croce CM. MicroRNA dysregulation in cancer: diagnostics, monitoring and therapeutics. A comprehensive review. EMBO Mol Med 2012; 4: 143-159.
31 Liu WH, Yeh SH, Lu CC, Yu SL, Chen HY, Lin CY et al. MicroRNA-18a prevents estrogen receptor-alpha expression, promoting proliferation of hepatocellular carcinoma cells. Gastroenterology 2009; 136: 683-693.

32 Pineau P, Volinia S, McJunkin K, Marchio A, Battiston C, Terris B et al. miR-221 overexpression contributes to liver tumorigenesis. Proc Natl Acad Sci USA 2010; 107: 264-269.

33 Gramantieri L, Fornari F, Ferracin M, Veronese A, Sabbioni S, Calin GA et al. MicroRNA-221 targets Bmf in hepatocellular carcinoma and correlates with tumor multifocality. Clin Cancer Res 2009; 15: 5073-5081.

34 Wong QW, Ching AK, Chan AW, Choy KW, To KF, Lai PB et al. MiR-222 overexpression confers cell migratory advantages in hepatocellular carcinoma through enhancing AKT signaling. Clin Cancer Res 2010; 16: $867-875$.

35 Tsai WC, Hsu PW, Lai TC, Chau GY, Lin CW, Chen CM et al. MicroRNA-122, a tumor suppressor microRNA that regulates intrahepatic metastasis of hepatocellular carcinoma. Hepatology 2009; 49: 1571-1582.

36 Xu T, Zhu Y, Xiong Y, Ge Y Y, Yun J P, Zhuang S M. MicroRNA-195 suppresses tumorigenicity and regulates G1/S transition of human hepatocellular carcinoma cells. Hepatology 2009; 50: 113-121.

37 Qu KZ, Zhang K, Li H, Afdhal NH, Albitar M. Circulating microRNAs as biomarkers for hepatocellular carcinoma. J Clin Gastroenterol 2011; 45: 355-360.

$38 \mathrm{Xu}$ J, Wu C, Che X, Wang L, Yu D, Zhang T et al. Circulating microRNAs, miR-21, miR-122, and miR-223, in patients with hepatocellular carcinoma or chronic hepatitis. Mol Carcinog 2011; 50: $136-142$.

39 Raposo G, Stoorvogel W. Extracellular vesicles: exosomes, microvesicles, and friends. J Cell Biol 2013; 200: 373-383.

40 Vlassov AV, Magdaleno S, Setterquist R, Conrad R. Exosomes: current knowledge of their composition, biological functions, and diagnostic and therapeutic potentials. Biochim Biophys Acta 2012; 1820: 940-948.

41 Tanaka Y, Kamohara H, Kinoshita K, Kurashige J, Ishimoto T, Iwatsuki M et al. Clinical impact of serum exosomal microRNA-21 as a clinical biomarker in human esophageal squamous cell carcinoma. Cancer 2013 119: 1159-1167.

42 Rabinowits G, Gercel-Taylor C, Day JM, Taylor DD, Kloecker GH. Exosomal microRNA: a diagnostic marker for lung cancer. Clin Lung Cancer 2009; 10: 42-46

$43 \mathrm{Ma}$ R, Jiang T, Kang X. Circulating microRNAs in cancer: origin, function and application. J Exp Clin Cancer Res 2012; 31: 38

$44 \mathrm{Li} \mathrm{J}$, Wang Y, Yu W, Chen J, Luo J. Expression of serum miR-221 in human hepatocellular carcinoma and its prognostic significance. Biochem Biophys Res Commun 2011; 406: 70-73.

45 Qi P, Cheng SQ, Wang H, Li N, Chen YF, Gao CF. Serum microRNAs as biomarkers for hepatocellular carcinoma in Chinese patients with chronic hepatitis B virus infection. PLoS ONE 2011; 6: e28486.

46 Sherman M. Hepatocellular carcinoma: epidemiology, surveillance, and diagnosis. Semin Liver Dis 2010; 30: 3-16.

47 Beasley RP, Hwang LY, Lin CC, Chien CS. Hepatocellular carcinoma and hepatitis B virus. A prospective study of 22707 men in Taiwan. Lancet 1981; 2: 1129-1133.

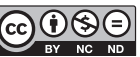

This work is licensed under a Creative Commons Attribution-NonCommercial-NoDerivs 4.0 International License. The images or other third party material in this article are included in the article's Creative Commons license, unless indicated otherwise in the credit line; if the material is not included under the Creative Commons license, users will need to obtain permission from the license holder to reproduce the material. To view a copy of this license, visit http://creativecommons.org/licenses/by-nc-nd/4.0/ 